\title{
A CHARACTERIZATION OF ODD ORDER EXTENSIONS OF THE FINITE PROJECTIVE SYMPLECTIC GROUPS $\operatorname{PSp}(4, q)$
}

BY

\author{
MORTON E. HARRIS( ${ }^{(1)}$
}

\begin{abstract}
In a recent paper, W. J. Wong characterized the finite projective symplectic groups $\operatorname{PSp}(4, q)$ where $q$ is a power of an odd prime integer by the structure of the centralizer of an involution in the center of a Sylow 2-subgroup of PSp $(4, q)$. In the present paper, finite groups which contain an involution in the center of a Sylow 2-subgroup whose centralizer has a more general structure than in the $\operatorname{PSp}(4, q)$ case are classified by showing them to be odd ordered extensions of $\operatorname{PSp}(4, q)$.
\end{abstract}

Introduction and statement. Let $p$ denote an odd prime integer and let $q=p^{f}$ where $f$ is a positive integer. Let $\operatorname{PSp}(4, q)$ denote the projective symplectic group in dimension 4 over a field $F_{q}$ of $q$ elements. The group PSp $(4, q)$ is described in $[8, \S 1]$, is simple of order $\frac{1}{2} q^{4}\left(q^{2}+1\right)\left(q^{2}-1\right)^{2}$ and has a Sylow 2-subgroup with center of order 2 so that involutions which lie in the centers of Sylow 2-subgroups form a single conjugacy class.

Let $\bar{\sigma}$ denote an automorphism of $F_{q}$. Then $\bar{\sigma}$ induces, in the natural way, an automorphism of PSp $(4, q)$ (cf. $[8, \S 1])$ and centralizes an involution in the center of a Sylow 2-subgroup of PSp $(4, q)$. In fact $\langle\bar{\sigma}\rangle$, the cyclic subgroup of Aut $\left(F_{q}\right)$ generated by $\bar{\sigma}$, acts faithfully on $\operatorname{PSp}(4, q)$ and one may form the natural semidirect product $\langle\bar{\sigma}\rangle \operatorname{PSp}(4, q)$. If $\bar{\sigma}$ is an odd ordered automorphism of $F_{q}$, then $\langle\bar{\sigma}\rangle \operatorname{PSp}(4, q)$ is an odd ordered extension of $\operatorname{PSp}(4, q)$ with trivial 2-core. In fact, any odd ordered extension of $\operatorname{PSp}(4, q)$ with trivial 2-core is of this form (cf. [1]).

Let $t$ be an involution in the center of a Sylow 2-subgroup of PSp $(4, q)$ such that $t$ is centralized by $\bar{\sigma}$. Then the centralizer $C(t)$ of $t$ in $\langle\bar{\sigma}\rangle \operatorname{PSp}(4, q)$ is a semidirect product $\langle\bar{\sigma}\rangle \mathscr{C}$ where $\mathscr{C}$ denotes the centralizer of $t$ in $\operatorname{PSp}(4, q)$ and $C(t)$ has trivial 2-core.

Finite groups $G$ containing an involution $t$ such that $C_{G}(t) \cong \mathscr{C}$ have been classified in [8]. However, for example in classifying groups by their Sylow 2-subgroups, one may arrive at a situation in which the centralizer $C_{G}(t)$ of an involution $t$ in a group

Received by the editors August 19, 1970 and, in revised form, February 15, 1971.

AMS 1970 subject classifications. Primary 20D05.

Key words and phrases. Centralizer of an involution, projective symplectic groups, simple group, odd ordered extension.

( $\left.{ }^{1}\right)$ This research was partially supported by National Science Foundation Grant GP-9584 at the University of Illinois at Chicago Circle. 
$G$ is an odd ordered extension of $\mathscr{C}$ with trivial 2-core. This is the case in the group $\langle\bar{\sigma}\rangle \operatorname{PSp}(4, q)$ of the above paragraph.

To handle this situation, we prove the following more general result:

THEOREM. Let $G$ be a finite group with an involution $t$ such that

(a) $O\left(C_{G}(t)\right)=\{1\}$ and

(b) $C_{G}(t)$ contains a normal subgroup $\mathfrak{S}$ of index $2 \rho$ with $\rho$ odd and an involution $u \in C_{G}(t)-\mathfrak{S}$ such that $\mathfrak{H}$ contains a subgroup $L_{1} \cong \operatorname{SL}(2, q)$ where $q=p^{f}, p$ is an odd prime integer and $f$ a positive integer such that if $L_{2}=L_{1}^{u}$, then $\mathfrak{Y}=L_{1} L_{2}$, $\left[L_{1}, L_{2}\right]=\{1\}$ and $L_{1} \cap L_{2}=\langle t\rangle$.

Then, either (i) $G=C_{G}(t) O(G)$ or (ii) $G \cong\langle\bar{\sigma}\rangle \operatorname{PSp}(4, q)$ where $\bar{\sigma} \in \operatorname{Aut}\left(F_{q}\right)$ and is of order $\rho$.

Note that when $p=1$, then $C_{G}(t)$ is isomorphic to the centralizer of an involution in the center of a Sylow 2-subgroup in $\operatorname{PSp}(4, q)$ and the theorem follows from [8].

Thus for the rest of the paper, we assume that $G$ is a finite group with an involution $t$ such that $C_{G}(t)$ satisfies the hypotheses of the theorem with $\rho>1$. Let $q \equiv \delta(\bmod 4)$ where $\delta= \pm 1$ and let $q-\delta=2^{n} e$ with $e$ an odd integer and $n \geqq 2$.

The paper is organized as follows. In $\S 1$, we study the structure of $C_{G}(t)$ and in $\S 2$, we show that if case (i) of the conclusion of the theorem does not hold, then $G$ has exactly two conjugacy classes of involutions. In $\S 3$, we determine the structure of the centralizer of an involution in the second conjugacy class, and we show that the situation is exactly as in the group $\langle\bar{\sigma}\rangle \operatorname{PSp}(4, q)$. Moreover, we show, using a lemma of Thompson, that $|G|=\rho|\operatorname{PSp}(4, q)|$. Then in $\S 4$, we find the structure of the normalizer of a Sylow $p$-subgroup of $G$. In $\S 5$, we construct a normal subgroup $G_{0}$ of $G$ such that $\left|G: G_{0}\right|=\rho$ and then we show that $G_{0}$ is isomorphic to PSp $(4, q)$ by applying [8]. The rest of the theorem now follows easily.

Our notation is fairly standard and tends to follow that of [5]. We use $O(X)$ to denote the 2-core of $X$, i.e., the largest normal subgroup of odd order in the finite group $X$. We write $[x, y]=x^{-1} x^{y}=x^{-1} y^{-1} x y$ and, if $x^{y}=z$, we write $y: x \rightarrow z$. If $y: x \rightarrow x^{-1}$, we say that $y$ inverts $x$ and, if $y: x \rightarrow z$ and $y: z \rightarrow x$, we write $y: x \leftrightarrow z$.

1. The structure of $C_{G}(t)$. Since $C_{G}(t) / \mathfrak{S}$ is a group of order $2 \rho$ we have

Lemma 1.1. $C_{G}(t)$ contains a subgroup $\mathscr{A}$ of index 2 such that $\mathscr{A} \geqq \mathfrak{S}$.

LeMma 1.2. $\left\{L_{1}, L_{2}\right\}$ is invariant in $C_{G}(t)$.

Proof. This follows easily from the Krull-Schmidt Theorem applied to the group $\mathfrak{K} /\langle t\rangle \cong \operatorname{PSL}(2, q) \times \operatorname{PSL}(2, q)$.

Corollary 1.2.1. $N_{G}\left(L_{1}\right)=N_{G}\left(L_{2}\right)=\mathscr{A}$.

Proof. Because $Z\left(L_{1}\right)=\langle t\rangle$, we have $N_{G}\left(L_{1}\right) \leqq C_{G}(t)$. Also $\mathfrak{S}_{\mathfrak{G}} \leqq N_{G}\left(L_{1}\right) \cap N_{G}\left(L_{1}\right)$ and $|\mathscr{A}| \mathfrak{S} \mid=\rho$ is odd. Now we can conclude that $\mathscr{A} \leqq N_{G}\left(L_{1}\right)$ from Lemma 1.2. Hence $N_{G}\left(L_{1}\right)=\mathscr{A}$. Similarly $N_{G}\left(L_{2}\right)=\mathscr{A}$. 
Lemma 1.3. $C_{G}(\mathfrak{S})=\langle t\rangle$.

Proof. Since $C_{G}(\mathfrak{S}) \leqq \mathscr{A}$ and $Z(\mathfrak{S})=\langle t\rangle, C_{G}(\mathfrak{S})$ has order $2 \cdot r$ where $r \mid \rho$. But $C_{G}(\mathfrak{S}) \triangleleft C_{G}(t)$ and $O\left(C_{G}(t)\right)=\{1\}$; hence $r=1$.

Lemma 1.4. There exists a subgroup $A$ of $\mathscr{A}$ of order $\rho$ such that $\mathscr{A}=\mathfrak{S} A$ and $A \cap \mathfrak{S}=\{1\}$ and such that $A$ centralizes a Sylow 2-subgroup of $\mathfrak{S}$. Moreover if, $f=2^{a}, a \geqq 0$, then $\rho=1$ and the result follows from [8].

Proof. Since $C_{\mathfrak{S}}\left(L_{1}\right)=L_{2} \triangleleft C_{\mathscr{A}}\left(L_{1}\right) \triangleleft \mathscr{A}$ and $\left|\mathscr{A}: L_{1} C_{\mathscr{A}}\left(L_{1}\right)\right|$ divides $\rho$, it follows from the structure of Aut $\left(L_{1}\right)$ that there exists a subgroup $A_{1}$ of $\mathscr{A}$ such that $A_{1} \geqq C_{\mathscr{A}}\left(L_{1}\right), \mathscr{A}=L_{1} A_{1}$, and $L_{1} \cap A_{1} \leqq C_{\mathscr{A}}\left(L_{1}\right)$. Since $\left|A_{1}: C_{\mathscr{A}}\left(L_{1}\right)\right|$ is odd, $A_{1}$ centralizes a Sylow 2-subgroup $S_{1}$ of $L_{1}$. Thus $\langle t\rangle \leqq L_{1} \cap A_{1} \leqq C_{\mathscr{A}}\left(L_{1}\right) \cap L_{1}=\langle t\rangle$ and $\mathfrak{S} \cap A_{1}=L_{2} \triangleleft A_{1}$. Hence $\left|A_{1} / L_{2}\right|$ is odd. Again, it follows that there exists a subgroup $A_{2}$ of $A_{1}$ such that $A_{2} \geqq C_{A_{1}}\left(L_{2}\right), A_{1}=L_{2} A_{2}$ and $L_{2} \cap A_{2} \leqq C_{A_{1}}\left(L_{2}\right)$ and such that $A_{2}$ centralizes a Sylow 2-subgroup $S_{2}$ of $L_{2}$. Thus $\langle t\rangle \leqq A_{2} \cap \mathfrak{F}_{2}=$ $A_{2} \cap A_{1} \cap \mathfrak{F}=A_{2} \cap L_{2} \leqq L_{2} \cap C_{A_{1}}\left(L_{2}\right)=\langle t\rangle$. Hence $\langle t\rangle$ is the Sylow 2-subgroup of $A_{2}$, and $A_{2}$ has a subgroup $A$ of index 2. Then $\mathfrak{S} A=L_{1} L_{2} A=L_{1} L_{2} A_{2}=L_{1} A_{1}=\mathscr{A}$, $\mathfrak{S} \cap A=A \cap \mathfrak{S} \cap A_{2}=A \cap\langle t\rangle=\{1\},|A|=\rho$ and $A$ centralizes $S_{1} S_{2}$ which is a Sylow 2-subgroup of $\mathfrak{S}$. Finally, if $f=2^{a}, a \geqq 0$ then

$$
\left|\mathscr{A}: L_{1} C_{\mathscr{A}}\left(L_{1}\right)\right| \text { and }\left|A_{1} / L_{2} C_{A_{1}}\left(L_{2}\right)\right|
$$

are powers of 2. Thus $A_{1}=C_{\mathscr{A}}\left(L_{1}\right), A_{2}=C_{A_{1}}\left(L_{2}\right)$ and $A_{2} \leqq C_{G}(\mathfrak{S})=\langle t\rangle$ and, therefore, $|A|=1=\rho$.

Note that we may now assume that $q>3$ and, hence, that $L_{i} /\langle t\rangle$ is simple for $i=1,2$. We may also assume that $e>1$, for if $e=1$ then $q-\delta=2^{n}$ and [6, Lemma 19.3] yields that either $q$ is a prime or $q=3^{2}$ both of which have been excluded.

LeMmA 1.5. A is Abelian with at most 2 generators.

Proof. Consider the natural homomorphism

$$
\theta: A \rightarrow\left(\operatorname{Aut}\left(L_{1}\right) / \operatorname{Inn}\left(L_{1}\right)\right) \times\left(\operatorname{Aut}\left(L_{2}\right) / \operatorname{Inn}\left(L_{2}\right)\right) .
$$

Suppose that $a \in \operatorname{Ker}(\theta)$. Then there is an $l_{i} \in L_{i}$ such that $a l_{i} \in C_{G}\left(L_{i}\right)$ for $i=1,2$. Hence $a l_{1} l_{2} \in C_{G}(\mathfrak{H})=\langle t\rangle$ and $a \in A \cap \mathfrak{S}_{c}=\{1\}$. Thus $\theta$ is one-to-one and since $|A|=\rho$ is odd, the lemma follows from the structures of Aut $\left(L_{1}\right)$ and Aut $\left(L_{2}\right)$.

LemMa 1.6. The subgroup $A$ may be chosen so that it is normalized by $u$. If $A$ is so chosen, there exists a Sylow 2-subgroup $S_{1}$ of $L_{1}$ centralized by $A$ and hence $S_{1}^{u}$ is a Sylow 2-subgroup of $L_{2}$ centralized by $A$.

Proof. Let $S_{1}$ be a Sylow 2-subgroup of $L_{1}$ centralized by $A$. Since $A$ centralizes a Sylow 2-subgroup of $L_{2}$ also, by conjugating $A$ by an element of $L_{2}$, we may assume that $A$ centralizes $S_{1} S_{1}^{u}$. Since $C_{G}\left(S_{1} S_{1}^{u}\right)=\langle t\rangle \times A$ and $u \in N_{G}\left(S_{1} S_{1}^{u}\right), u$ normalizes $O\left(C_{G}\left(S_{1} S_{1}^{u}\right)\right)=A$.

Henceforth, we assume that $A$ is normalized by $u$. 
Corollary 1.6.1. $A=C_{A}(u) \times[A, u]$ where $[A, u]$ is the subgroup of $A$ formed by the elements of $A$ inverted by $u$ together with the identity element.

Let

$$
B=\left\{x_{1} x_{1}^{u} \mid x_{1} \in L_{1}\right\}
$$

Then $B$ is a subgroup of $C_{G}(t, u)$ and $B$ is isomorphic to PSL $(2, q)$ where the isomorphism is induced by the epimorphism $\theta: L_{1} \rightarrow B$ defined by $\theta\left(x_{1}\right)=x_{1} x_{1}^{u}$ for $x_{1} \in L_{1}$ since $\operatorname{Ker}(\theta)=\langle t\rangle$.

An easy calculation gives

LEMmA 1.7. $C_{G}(u, t)=(B \times\langle t, u\rangle) C_{A}(u)$ where $\mathfrak{S} \cap C_{G}(u, t)=(B \times\langle t\rangle) \triangleleft C_{G}(u, t)$.

Corollary 1.7.1. B is characteristic in $C_{G}(u, t)$.

Proof. Since $q>3, B$ is simple and non-Abelian, thus $C_{G}(u, t)^{\prime \prime}=B$.

2. Classes of involutions in $G$. From the structure of $\mathscr{H}$, we have

LeMma 2.1. All involutions of $\mathfrak{S}-\langle t\rangle=L_{1} L_{2}-\langle t\rangle$ are conjugate in $\mathfrak{g}=L_{1} L_{2}$.

An easy consequence of the fact that $u$ normalizes $A$ is

LEMma 2.2. All involutions of $C_{G}(t)-\mathfrak{S}$ are conjugate in $C_{G}(t)$ to $u$ or $t u$.

The proof of $[2,(2 \mathrm{~B})]$ yields

LEMMA 2.3. If $H$ is a subgroup of $G$ and if $T$ is a Sylow 2-subgroup of $H \cap C_{G}(t)$ such that $\langle t\rangle$ is characteristic in $T$, then $T$ is a Sylow 2-subgroup of $H$. In particular, a Sylow 2-subgroup of $C_{G}(t)$ is a Sylow 2-subgroup of $G$.

Let $S_{1}=\left\langle a_{1}, b_{1}\right\rangle$ where $a_{1}^{2^{n-1}}=b_{1}^{2}=t$ and $a_{1} b_{1}=b_{1} a_{1}^{-1}$ be a Sylow 2-subgroup of $L_{1}$ centralized by $A$. Set $b_{2}=b_{1}^{u}, a_{2}=a_{1}^{u}$. Then $v=\left(a_{1} a_{2}\right)^{2^{n-2}}$ and $w=b_{1} b_{2}$ are two involutions in $\mathfrak{F}-\langle t\rangle$ centralized by $u$. Set $S=S_{1} S_{1}^{u}\langle u\rangle=\left\langle a_{1}, b_{1}, a_{2}, b_{2}, u\right\rangle$. Then $S$ is a Sylow 2-subgroup of $G$. Note that $Z(S)=\langle t\rangle$ so that the conjugacy class of $t$ in $G$ consists exactly of those involutions which are in the center of some Sylow 2-subgroup of $G$.

Lemma 2.4. The involutions of $\mathfrak{S}-\langle t\rangle$ are not conjugate in $G$ to $t$.

Proof. It suffices to show that $v$ is not conjugate in $G$ to $t$. Since $C_{L_{1}}\left(a_{1}^{2^{n-2}}\right)=\left\langle d_{1}\right\rangle$ which is cyclic of order $q-\delta$, we may assume that $d_{1}^{e}=a_{1}$. Setting $d_{2}=d_{1}^{u}$, we have

$$
C_{G}(t, v)=\left\langle d_{1}, d_{2}, w\right\rangle A \cdot\langle u\rangle
$$

which is of order $2(q-\delta)^{2} \rho$. Also $A$ normalizes $\left\langle d_{1}\right\rangle$ and $\left\langle d_{2}\right\rangle$ and $d_{i}^{w}=d_{i}^{-1}$ for $i=1$, 2. A Sylow 2-subgroup of $C_{G}(t, v)$ is $T=\left\langle a_{1}, a_{2}, w, u\right\rangle$ which is of order $2^{2 n+1}$. The argument of [8, Lemma 2.2] applies with only a slight modification required if $n=2$. 
We now assume

$$
G \neq C_{G}(t) O(G)
$$

The proof of $[8$, Lemma 2.3] yields

LEMMA 2.5. Either $t$ or tu is conjugate in $G$ to $t$.

Since $C_{G}(t)$ has an automorphism which interchanges $u$ and $t u$ and fixes $\mathfrak{F} A$, we may assume

$$
t u \text { is conjugate in } G \text { to } t \text {. }
$$

LEMMA 2.6. G has exactly two conjugacy classes of involutions $K_{1}$ and $K_{2}$ such that $K_{1} \cap C_{G}(t)$ consists of the conjugacy classes in $C_{G}(t)$ represented by $t$ and $t u$ and $K_{2} \cap C_{G}(t)$ consists of the conjugacy classes in $C_{G}(t)$ represented by $v$ and $u$. There exists an element $z \in N_{G}\left(C_{S}(t u)\right)$ such that $z^{2} \in C_{S}(t u)$ and

$$
z: t \rightarrow u v, \quad u \rightarrow t v, \quad v \rightarrow v .
$$

Also z normalizes $C_{A}(u)$.

Proof. Let $K_{1}$ be the conjugacy class of $t$ in $G$ and let $K_{2}$ be the conjugacy class of $v$ in $G$; clearly $K_{1} \neq K_{2}$ by Lemma 2.4 . Set

$$
E=C_{S}(t u)=\left\langle a_{1} a_{2}, w\right\rangle \times\langle t, u\rangle .
$$

Then $C_{G}(E)=Z(E) \times C_{A}(u)$ by Lemma 1.7 and the argument of [8, Lemma 2.4] applies with only a slight modification required if $n=2$.

The discussion at the end of $[8, \S 2]$ yields

Lemma 2.7. The focal subgroup of $S$ in $G$ is $S$ and hence $G$ has no subgroup of index 2.

\section{Centralizers of involutions in $K_{2}$. Clearly}

$$
C_{G}(t, v)=\left\langle d_{1}, d_{2}, w\right\rangle A\langle t u\rangle .
$$

Transforming by the element $z$ of Lemma 2.6, we obtain

$$
C_{G}(u, v)=\left\langle d_{1}^{z}, d_{2}^{z}, w^{z}\right\rangle A^{z}\langle t u\rangle .
$$

LEMMA 3.1. $\left\langle d_{1} d_{2}\right\rangle^{z}=\left\langle d_{1} d_{2}\right\rangle,\left\langle a_{1} a_{2}\right\rangle^{z}=\left\langle a_{1} a_{2}\right\rangle$ and

$$
w=\left(w\left(d_{1} d_{2}\right)^{m} t u\right)^{z} \quad \text { for some integer } m .
$$

Proof. Since $C_{G}(t, u, v)=\left(\langle t, u\rangle \times\left\langle d_{1} d_{2}, w\right\rangle\right) C_{A}(u)$ by Lemma 1.7 and since $\left\langle d_{1} d_{2}, w\right\rangle$ is dihedral of order $q-\delta$, we have

$$
O^{2 \prime}\left(C_{G}(t, u, v)\right)=\langle t, u\rangle \times\left\langle d_{1} d_{2}, w\right\rangle .
$$

Since $z \in N_{G}(\langle t, u, v\rangle)$, we obtain

$$
O^{2 \prime}\left(C_{G}(t, u, v)\right)=\langle t, u\rangle \times\left\langle\left(d_{1} d_{2}\right)^{z}, w^{z}\right\rangle .
$$


Clearly the proof of $\left[8\right.$, Lemma 3.1] applies with $C_{G}(t, u, v)$ replaced by

$$
O^{2 \prime}\left(C_{G}(t, u, v)\right) \text {. }
$$

Since $C_{G}(u, v)=\left\langle d_{1}^{z}, d_{2}^{z}, w^{z}\right\rangle A^{z}\langle t u\rangle$ by (8), $\left\langle t u, a_{1}^{z}, a_{2}^{z}\right\rangle$ is a subgroup of $C_{G}(u, v)$ of order $2^{2 n}$. Also $w$ centralizes $t u$ and, by (9), we have $\left(a_{1}^{z}\right)^{w}=\left(a_{2}^{z}\right)^{-1},\left(a_{2}^{z}\right)^{w}=\left(a_{1}^{z}\right)^{-1}$. Thus $C_{G}(u, v)$ contains the subgroup $T=\left\langle t u, a_{1}^{z}, a_{2}^{z}, w\right\rangle$ of order $2^{2 n+1}$. Since $u$ does not lie in the center of a Sylow 2-subgroup of $G, T$ is a Sylow 2-subgroup of $C_{G}(u), C_{G}(v)$ and $C_{G}(u, v)$.

LEMMA 3.2. $C_{G}(u)$ has a normal subgroup $K$ of index 2 with Sylow 2-subgroup $M=\left\langle a_{1}^{z}, a_{2}^{z}, w\right\rangle$. Also $C_{G}(u)=K\langle t\rangle$ where $K \cap\langle t\rangle=\{1\}$.

Proof. The proof of [8, Lemma 3.2] applies to demonstrate the existence of $K$. Since $u=(t v)^{z}=\left(a_{1}^{2^{n-1}}\right)^{z}\left(\left(a_{1} a_{2}\right)^{2^{n-2}}\right)^{z} \in M$ and $t \notin K$, the result follows.

Lemma 3.3. $K$ has a normal subgroup $L$ of index $2^{n}$ with Sylow 2-subgroup $J=\left\langle a_{1} a_{2}, w\right\rangle$.

Proof. Clearly $M^{\prime}=\left\langle\left(a_{1} a_{2}\right)^{z}\right\rangle=\left\langle a_{1} a_{2}\right\rangle \quad$ and $\quad$ so $\quad N_{K}(M) \leqq C_{K}(v) \leqq C_{G}(u, v)=$ $\left\langle d_{1}^{z}, d_{2}^{z}, w^{z}\right\rangle A^{z}\langle t u\rangle$ which has $\left\langle\left(d_{1}^{2^{n}}\right)^{z},\left(d_{2}^{2^{n}}\right)^{z}\right\rangle A^{z}$ as a normal 2-complement. Hence $N_{K}(M)$ has a normal 2-complement so that $N_{K}(M)^{\prime} \cap M=M^{\prime}$. With only a slight modification, the proof of $[8$, Lemma 3.4] yields the result.

Since $u \notin L$, we have

LEMMA 3.4. If $k$ is any integer and $s=a_{1}^{z} w\left(d_{1} d_{2}\right)^{k}$, then $s^{2^{n-1}}=u$, the order of $s$ is $2^{n}, s^{t}=s^{-1}$ and hence $\langle t, s\rangle$ is dihedral of order $2^{n+1}$. Also $K=L\langle s\rangle$ and $L \cap\langle s\rangle=\{1\}$.

LemmA 3.5. (a) For $i=1$ or 2 , if $x \in N_{G}\left(L_{i}\right)=\mathscr{A}$ and $x$ acts trivially on $L_{i} /\langle t\rangle$ then $x$ centralizes $L_{i}$.

(b) If $x \in C_{G}(B) \cap \mathscr{A}$, then $x \in\langle t\rangle$.

Proof. Let $x$ satisfy the hypotheses of (a) and let $y \in L_{i} \cong \operatorname{SL}(2, q), y$ of odd order.

Clearly $x$ centralizes $y^{2}$ and hence $[x, y]=1$. But $L_{i}=\operatorname{SL}(2, q)$ is generated by its elements of odd order and (a) follows. Let $x$ satisfy the hypothesis of (b) and let $y_{1} \in L_{1}$. Then $\left(y_{1} y_{1}^{u}\right)^{x}=y_{1} y_{1}^{u}=y_{1}^{x} y_{1}^{u x}$, so that $y_{1}^{-1} y_{1}^{x} \in L_{1} \cap L_{2}=\langle t\rangle$. Hence $x$ acts trivially on $L_{1} /\langle t\rangle$ and we conclude from (a) that $x$ centralizes $L_{1}$. Similarly $x$ centralizes $L_{2}$. Thus $x \in\langle t\rangle$ by Lemma 1.3.

We shall need the following well-known result:

LEMMA 3.6. Let $H$ be a group with $O(H)=\{1\}$ and such that $H$ contains a normal subgroup $M \cong \operatorname{PSL}(2, r)$ with $r$ a prime power. Suppose also that $4 \nmid|H: M|$. Then $H / M$ is Abelian.

LemmA 3.7. (a) $u$ centralizes $A$ and hence $A \leqq L$.

(b) $B$ is characteristic in $L$.

(c) $L=(B \times O(L)) A$ and $(B \times O(L)) \cap A=\{1\}$. 
Proof. $A^{z} \leqq L$ and $B \leqq L$ since $B$ is simple. Also $B \cap O(L)=\{1\}$ and $z \in N_{G}\left(C_{A}(u)\right.$ ). Moreover, $C_{G}(t, u)=(B \times\langle t, u\rangle) C_{A}(u)$ and $\mathfrak{S} \cap C_{G}(t, u)=B \times\langle t\rangle$ so that $C_{A}(u)$ normalizes $B$. If $a \in C_{A}(u)$ centralizes $B$, then $a \in\langle t\rangle$ by Lemma 3.5(b). Hence $a=1$ and $C_{A}(u)$ acts faithfully on $B$. But $\left[O(L) \cap C_{A}(u), B\right] \leqq B \cap O(L)=\{1\}$. Thus $O(L) \cap C_{A}(u)=\{1\}$. By a theorem of Gorenstein and Walter [4, Theorem 1], since $J$ is dihedral and $B \cong B O(L) / O(L)$ is an odd indexed subgroup of $L / O(L)$, we have two cases:

(a) $L / O(L)$ is isomorphic to the alternating group $\mathscr{A}_{7}$. Hence $B$ is isomorphic to a subgroup of $\mathscr{A}_{7}$ which implies that $q=5,7$ or 9 . These possibilities have been excluded.

(b) $L / O(L)$ is isomorphic to a subgroup of P PL $(2, r)$ containing PSL $(2, r)$ with $r$ odd. Clearly $B$ must be isomorphic to a subgroup of PSL $(2, r)$ so that $q=5$ (which is excluded) or $r$ is a power of $q$. Let $M$ be the subgroup of $L$ such that $L \unrhd M>O(L)$ and $M / O(L) \cong \operatorname{PSL}(2, r)$. Thus $B O(L) \leqq M$ and $|L: M|$ is odd. Now $C_{G}(u, v)=\left\langle d_{1}^{z}, d_{2}^{z}, w^{z}\right\rangle A^{z}\langle t u\rangle \geqq\langle s, t\rangle$. But $C_{G}(u)=L\langle s, t\rangle$; hence $C_{G}(u, v)=C_{L}(v)\langle s, t\rangle$. Clearly $C_{L}(v) \geqq\left\langle d_{1}^{2^{n} z}, d_{1} d_{2}, w\right\rangle \cdot A^{z}$ which is a product of two groups. But $\left|C_{G}(t, v)\right|$ $=\left|C_{G}(u, v)\right|=2(q-\delta)^{2} \cdot \rho$ so that $\left|C_{L}(v)\right|=2^{n} e^{2} \rho$ (for $L \cap\langle s, t\rangle=\{1\}$ ). Now $\left\langle d_{1}^{2^{n} z}, d_{1} d_{2}, w\right\rangle$ has a normal 2-complement, namely, $\left\langle d_{1}^{2^{n_{z}}},\left(d_{1} d_{2}\right)^{2^{n-1}}\right\rangle$ and

$$
\begin{aligned}
\left\langle d_{1}^{2^{2} z},\left(d_{1} d_{2}\right)^{2^{n-1}}\right\rangle \cap A^{z} & =\left\langle d_{1}^{2^{n} z}\left(d_{1} d_{2}\right)^{2^{n-1} z}\right\rangle \cap A^{z} \\
& =\left(\left\langle d_{1}^{2^{n}},\left(d_{1} d_{2}\right)^{2^{n-1}}\right\rangle \cap A\right)^{z}=\{1\} .
\end{aligned}
$$

Thus $\left|\left\langle d_{1}^{2^{n} z}, d_{1} d_{2}, w\right\rangle \cdot A^{z}\right|=2^{n} e^{2} \rho=\left|C_{L}(v)\right|$ and so $C_{L}(v)=\left\langle d_{1}^{2^{n_{z}}}, d_{1} d_{2}, w\right\rangle A^{z}$.

Clearly $L=O^{2}\left(C_{G}(u)\right)$.

Now $M / O(L)=(L / O(L))^{\prime}$ implies that $M=L^{\prime} O(L) \triangleleft C_{G}(u)$. Moreover $L \cap\langle t\rangle$ $=\{1\}$ and $L\langle t\rangle$ is a subgroup of $C_{G}(u)$ such that $O(L\langle t\rangle)=O(L)$ and $L\langle t\rangle / O(L)$ contains a normal subgroup $M / O(L)$ of index twice an odd integer. Now Lemma 3.6 applies, and we conclude that $L\langle t\rangle / M$ is Abelian. Hence $\left[d_{1}^{2^{n_{z}}}, u^{z}\right]=\left[d_{1}^{2^{n} z}, t v\right]$ $=d_{1}^{-2^{2} z} d_{2}^{2^{n} z}=\left(d_{1}^{-1} d_{2}\right)^{2^{n} z} \in M$; but $\left(d_{1}^{-1} d_{2}\right)^{2^{n-1}}$ has order $e$, therefore $\left(d_{1}^{-1} d_{2}\right)^{2^{n-1} z} \in M$. Suppose that $w=w^{z}\left(d_{1} d_{2}\right)^{m z} t u \in \mathfrak{S}^{z} A^{z}$. Then $w \in \mathfrak{S}^{z}, t u \in \mathfrak{S g}^{z}$ and $t u \in \mathfrak{F}$ which is impossible. Thus $C_{L}(v) \cap \mathfrak{S}^{z} A^{z}=\left\langle d_{1}^{2^{n_{z}}}, d_{1} d_{2}\right\rangle A^{z}$ and $C_{L}(v) \cap \mathfrak{S}^{z}=\left\langle d_{1}^{2^{n_{z}}}, d_{1} d_{2}\right\rangle$ $=\left\langle\left(d_{1} d_{2}^{-1}\right)^{2^{n-1} z}, d_{1} d_{2}\right\rangle$ is normalized by $A^{z}$. Also

$$
C_{M}(v)=\left\langle\left(d_{1} d_{2}^{-1}\right)^{2^{n-1} z}, d_{1} d_{2}, w\right\rangle\left(A^{z} \cap M\right)
$$

is normalized by $A^{z}$ and $C_{M}(v, w) \geqq\left\langle\left(d_{1} d_{2}^{-1}\right)^{2^{n-1}}\right\rangle \times\langle v, w\rangle$. However,

$$
C_{M / O(L)}(\langle v, w\rangle O(L))=\langle v, w\rangle O(L) / O(L)
$$

since $M / O(L)=\operatorname{PSL}(2, r)$. Thus $\left(d_{1} d_{2}^{-1}\right)^{2^{n-1} z} \in O(L)$ and $A^{z}$ normalizes $C_{L}(v) \cap \mathfrak{S}^{z}$ $\cap O(L)=\left\langle\left(d_{1} d_{2}^{-1}\right)^{2^{n-1} z}\right\rangle$. Thus $A$ normalizes $\left\langle\left(d_{1} d_{2}^{-1}\right)^{2^{n-1}}\right\rangle=\left\langle\left(d_{1} d_{2}^{-1}\right)^{2^{n}}\right\rangle$ which is a group of order $e>1$. In the proof of Lemma 2.4, we saw that $A$ normalizes both $\left\langle d_{1}\right\rangle$ and $\left\langle d_{2}\right\rangle$. Hence $A$ normalizes $\left\langle d_{1}^{2^{n}}\right\rangle$ and $\left\langle d_{2}^{2^{n}}\right\rangle$ which are groups of order $e>1$. Let $a \in[A, u]$ and let $\left(\left(d_{1} d_{2}^{-1}\right)^{2^{n}}\right)^{a}=\left(d_{1} d_{2}^{-1}\right)^{2^{n_{\gamma}}}=d_{1}^{2^{n_{\gamma}}} d_{2}^{-2^{n_{\gamma}}}$ where $\gamma$ is an integer relatively prime to $e$. Let $d_{1}^{2^{n} a}=d_{1}^{2^{n} \alpha}$ where $\alpha$ is an integer relatively prime to 
$e$. Then $d_{2}^{2^{n} a}=d_{1}^{2^{n} u a}=d_{1}^{2^{n} a^{-1} u}=d_{1}^{2^{2 n} \beta u}=d_{2}^{2^{n} \beta}$ where $\beta$ is an integer relatively prime to $e$ such that $\alpha \beta \equiv 1(\bmod e)$ (since $u$ inverts $a)$. Hence $\left(d_{1} d_{2}^{-1}\right)^{2^{n} a}=d_{1}^{2^{n} \gamma} d_{2}^{-2^{n_{\gamma}}}=$ $d_{1}^{2^{n} \alpha} d_{2}^{-2^{n} \beta}$. Thus $\alpha \equiv \beta(\bmod e)$ and $\alpha^{2} \equiv 1(\bmod e)$. Since $a$ is of odd order, $a$ centralizes $d_{1}^{2^{n}}$ and $d_{2}^{2^{n}}$. Consequently $a$ centralizes $\left\langle a_{1}\right\rangle \times\left\langle d_{1}^{2^{n}}\right\rangle=\left\langle d_{1}\right\rangle$ and similarly for $\left\langle d_{2}\right\rangle$. But a subgroup of order $q-\delta$ contained in SL $(2, q)$ is self-centralizing in Aut (SL $(2, q))$; thus there exist elements $d_{1}^{\prime} \in\left\langle d_{1}\right\rangle$ and $d_{2}^{\prime} \in\left\langle d_{2}\right\rangle$ such that $d_{1}^{\prime} d_{2}^{\prime} a$ centralizes $L_{1} L_{2}=\mathfrak{H}$. Hence $d_{1}^{\prime} d_{2}^{\prime} a \in C_{G}(\mathfrak{S})=\langle t\rangle$ and $a \in \mathfrak{S} \cap A=\{1\}$. We have proved that $[A, u]=1$ and thus $u$ centralizes $A$ and $z$ normalizes $A=C_{A}(u)$. Hence $C_{L}(v, w)=\left(\left\langle\left(d_{1} d_{2}^{-1}\right)^{2^{n-1} z}\right\rangle \times\langle v, w\rangle\right) A$ has a normal 2-complement $\left\langle\left(d_{1} d_{2}^{-1}\right)^{2^{n-1} z}\right\rangle A$ and $C_{M}(v, w)=\left(\left\langle\left(d_{1} d_{2}^{-1}\right)^{2^{n-1} z}\right\rangle \times\langle v, w\rangle\right)(M \cap A)$. Again $C_{M / O(L)}(\langle v, w\rangle O(L))=$ $\langle v, w\rangle O(L)$ and $M \cap A \leqq O(L)$ so that $M \cap A=\{1\}$. Consequently $C_{M}(v)=$ $\left\langle\left(d_{1} d_{2}^{-1}\right)^{2^{n-1} z}, d_{1} d_{2}, w\right\rangle$. Since $C_{M / O(L)}(v O(L))=C_{M}(v) O(L) / O(L)$, taking orders, we conclude that $r \pm 1=q-\delta$ (because $\left.O(L) \cap\left\langle\left(d_{1} d_{2}^{-1}\right)^{2^{n-1} z}, d_{1} d_{2}, w\right\rangle=\left\langle\left(d_{1} d_{2}^{-1}\right)^{2^{n-1} z}\right\rangle\right)$. But $r$ is a power of $q$; thus $q=r$ and $M=B O(L)$. Since $\left|C_{M}(v)\right|=e(q-\delta)=d^{2} 2^{n}$, $\left|C_{M}(v) \cap O(L)\right|=e$ and $w, v w$ are conjugate to $v$ in $B$, we also have $\left|C_{O(L)}(w)\right|$ $=\left|C_{O(L)}(w)\right|=e$. But $\left|O(L) \cap C_{M}(v, w)\right|=e$, and we may apply [3, Lemma 3] to conclude that $|O(L)|=e$. Thus $O(L)=\left\langle\left(d_{1} d_{2}^{-1}\right)^{2^{n-1} z}\right\rangle$. Since $C_{M}(O(L)) \triangleleft M$, $C_{M}(O(L))$ contains $\langle O(L), v\rangle$ and $M / O(L)$ is simple, we conclude that $C_{M}(O(L))=M$; thus $M=B \times O(L)$. We have $B=M^{\prime}$ so that (b) follows. Also all of the involutions of $B$ are conjugate in $B$ and $|L / B|$ is odd. Hence $\left|C_{L}(v): C_{M}(v)\right|=|L: M|=|A|$ and $L=$ $(B \times O(L)) A$ where $(B \times O(L)) \cap A=M \cap A=\{1\}$. This finishes the proof of the lemma.

Corollary 3.7.1. $O^{2 \prime}\left(C_{G}(t)\right)=\mathfrak{S}\langle u\rangle$.

Lemma 3.5(b) yields

LEMMA 3.8. $C_{A B}(B)=\{1\}$.

Consequently $A B$ acts faithfully by conjugation on $B$, and we have an injection $\theta: A B=B A \rightarrow$ Aut $(B)$ such that $\theta$ maps $B$ onto Inn $(B)$. Thus

$$
|A B|=\rho|B||| \text { Aut }(B)|=2| B \mid f
$$

so that $\rho$ divides $f$. Moreover, the image of $\theta, \operatorname{Im}(\theta)$, is the unique subgroup of Aut $(B)$ which contains $\operatorname{Inn}(B)$ as a subgroup of index $\rho$. (This follows from the structure of Aut $(B)$ and the fact that $\rho$ is odd.) Also $A \cong A B / B$ must be cyclic.

Let $F_{q}$ denote the field of $q=p^{f}$ elements and let SL $\left(2, F_{q}\right)$ denote the multiplicative group of $2 \times 2$ matrices with coefficients in $F_{q}$ of determinant 1 . Fix an isomorphism

$$
\phi_{1}: \operatorname{SL}\left(2, F_{q}\right) \rightarrow L_{1} .
$$

Let

$$
\phi_{2}=I_{u} \circ \phi_{1}: \operatorname{SL}\left(2, F_{q}\right) \rightarrow L_{2}
$$


where $I_{u}$ denotes conjugation by $u$. Clearly

$$
\phi_{1}\left(\left[\begin{array}{rr}
-1 & 0 \\
0 & -1
\end{array}\right]\right)=t=\phi_{2}\left(\left[\begin{array}{rr}
-1 & 0 \\
0 & -1
\end{array}\right]\right)
$$

Thus we have the obvious isomorphism

$$
\phi: \operatorname{PSL}\left(2, F_{q}\right)=\operatorname{SL}\left(2, F_{q}\right) /\left\langle\left[\begin{array}{rr}
-1 & 0 \\
0 & -1
\end{array}\right]\right\rangle \rightarrow B
$$

defined by:

$$
\text { if } x \in \operatorname{SL}\left(2, F_{q}\right) \text {, then } \phi\left(x\left\langle\left[\begin{array}{rr}
1 & 0 \\
0 & -1
\end{array}\right]\right\rangle\right)=\phi_{1}(x) \phi_{2}(x) \text {. }
$$

Since $\rho \mid f$, we may let $f=\rho f_{1}$ where $f_{1}$ is a positive integer. Let $\bar{\sigma}$ denote the automorphism of $F_{q}$ of order $\rho$ defined by

$$
\bar{\sigma}(x)=x^{p f_{1}} \quad \text { for } x \in F_{q} .
$$

Then $\bar{\sigma}$ induces an automorphism of order $\rho$ of $\operatorname{SL}\left(2, F_{q}\right)$ and of PSL $\left(2, F_{q}\right)$ $=\operatorname{SL}\left(2, F_{q}\right) /\left\langle\left[\begin{array}{rr}-1 & -0 \\ 0 & -1\end{array}\right]\right\rangle$. Since the natural semidirect product $\langle\bar{\sigma}\rangle \operatorname{Inn}\left(\operatorname{PSL}\left(2, F_{q}\right)\right)$ is the unique subgroup of Aut (PSL $\left(2, F_{q}\right)$ ) which contains Inn (PSL $\left(2, F_{q}\right)$ ) of index $\rho$, it follows from the above discussion of the subgroup $A B$ that there exists an element $\sigma \in A B$ of order $\rho$ such that $A B=\langle\sigma\rangle B, B \cap\langle\sigma\rangle=\{1\}$ and

$$
\phi\left(x\left\langle\left[\begin{array}{rr}
-1 & 0 \\
0 & -1
\end{array}\right]\right\rangle\right)^{\sigma}=\phi\left(x^{\bar{\sigma}}\left\langle\left[\begin{array}{rr}
-1 & 0 \\
0 & -1
\end{array}\right]\right\rangle\right) \text { for all } x \in \operatorname{SL}\left(2, F_{q}\right) \text {. }
$$

But $A B \leqq N_{G}\left(L_{1}\right) \cap N_{G}\left(L_{2}\right)$ so that $\phi_{1}(x)^{\sigma} \phi_{2}(x)^{\sigma}=\phi_{1}\left(x^{\bar{\sigma}}\right) \phi_{2}\left(x^{\bar{\sigma}}\right)$. Thus $\phi_{1}(x)^{-\sigma} \phi_{1}\left(x^{\bar{\sigma}}\right)$ $\in L_{1} \cap L_{2}=\langle t\rangle$ for all $x \in \operatorname{SL}\left(2, F_{q}\right)$. It follows as in the proof of Lemma 3.5(a) that

$$
\phi_{i}(x)^{\sigma}=\phi_{i}\left(x^{\bar{\sigma}}\right) \text { for } i=1,2 \text { and all } x \in \operatorname{SL}\left(2, F_{q}\right) .
$$

Moreover, $\mathfrak{S} \cap(A B)=B=\mathfrak{S} \cap(\langle\sigma\rangle B)$ implies that $\mathfrak{S} \cap\langle\sigma\rangle=1$ and

$$
(B \times O(L)) \cap(A B)=B=(B \times O(L)) \cap(\langle\sigma\rangle B)
$$

implies that $(B \times O(L)) \cap\langle\sigma\rangle=\{1\}$. It follows that we may replace $A$ by $\langle\sigma\rangle$, consequently from now on, we will assume

$$
A=\langle\sigma\rangle .
$$

If $\delta=1$, let $\varepsilon$ denote a generator of the multiplicative group $F_{q}^{\times}$of $F_{q}$ and set

$$
d=\left[\begin{array}{ll}
\varepsilon & 0 \\
0 & \varepsilon^{-1}
\end{array}\right] \text { and } b=\left[\begin{array}{rr}
0 & -1 \\
1 & 0
\end{array}\right]
$$

If $\delta=-1$, note that $f$ is odd and $p \equiv-1(\bmod 4)$ also. Let $\varepsilon$ denote a generator of the multiplicative group $F_{p}^{\times}$of $F_{p}$. Since $F_{q}^{\times}$has no element of order $4, \sqrt{ } \varepsilon \notin F_{q}$ so that, using the norm from $F_{p^{2}}^{\times} \rightarrow F_{p}{ }^{\times}$, there exist elements $\lambda, \mu \in F_{p}$ such that 
$\lambda^{2}-\varepsilon \mu^{2}=-1$ and, using the norm from $F_{q^{2}} \rightarrow F_{q}$, there exist elements $\alpha, \beta \in F_{q}$ such that $\alpha+\beta \sqrt{ } \varepsilon$ is a generator of the subgroup of elements of $F_{q^{2}}^{\times}$of norm 1. Set

$$
d=\left[\begin{array}{ll}
\alpha & \beta \\
\varepsilon \beta & \alpha
\end{array}\right] \text { and } b=\left[\begin{array}{cc}
\lambda & \mu \\
-\varepsilon \mu & -\lambda
\end{array}\right]
$$

Thus $b, d \in \operatorname{SL}\left(2, F_{q}\right)$ and it is easy to see that $\bar{\sigma}$ centralizes $b$ in both cases and $d^{\tilde{\sigma}}=d^{p_{1}}$ if $\delta=1$ and $d^{\tilde{\sigma}}=d^{-p^{f_{1}}}$ if $\delta=-1$. Moreover, $b^{2}=-I$ and $b$ inverts $d$. By (16), we have $\phi_{i}(b)^{\sigma}=\phi_{i}(b)$ for $i=1,2$ and $\phi_{i}(d)^{\sigma}=\phi_{i}(d)^{\delta f_{1}}$ for $i=1$, 2. Hence $\phi_{i}\left(d^{e}\right)^{\sigma}=\phi_{i}(d)^{\delta e f_{1}}$ and since $p^{f_{1}} \equiv \delta\left(\bmod 2^{n}\right)$ we have $\phi_{i}\left(d^{e}\right)^{\sigma}=\left(\phi_{i}(d)^{e}\right)^{\sigma}=\phi_{i}(d)^{e}$. Now note that we may assume

$$
b_{i}=\phi_{i}(b), \quad a_{i}=\phi_{i}(d)^{e}, \quad d_{i}=\phi_{i}(d) \quad \text { for } i=1,2 .
$$

LEMMA 3.9. For a suitable integer $k$ and $s=a_{1}^{z} w\left(d_{1} d_{2}\right)^{k}$, we have

$$
C_{G}(u)=L\langle s, t\rangle=((B \times O(L))\langle\sigma\rangle)\langle s, t\rangle
$$

where $B \cong \operatorname{PSL}(2, q), O(L)$ is cyclic of order $e$, and $\langle t, s\rangle$ is dihedral of order $2^{n+1}$ where $t^{2}=s^{2^{n}}=1, s^{t}=s^{-1}$ and $s^{2^{n-1}}=u$. Also $B, O(L), L=(B \times O(L))\langle\sigma\rangle$ and $(B \times O(L))\langle s, t\rangle$ are normal in $C_{G}(u)$ and $O^{2 \prime}\left(C_{G}(u)\right)=(B \times O(L))\langle s, t\rangle$. The involution $t$ centralizes $B$ and inverts $O(L)$; the element $s$ centralizes $O(L)$ and for any

$$
x \in \operatorname{SL}\left(2, F_{q}\right)
$$

we have

$$
\phi\left(x\left\langle\left[\begin{array}{rr}
-1 & 0 \\
0 & -1
\end{array}\right]\right\rangle\right)^{s}=\phi\left(x^{s}\left\langle\left[\begin{array}{rr}
-1 & 0 \\
0 & -1
\end{array}\right]\right\rangle\right)
$$

where $\bar{s}=\left[\begin{array}{cc}0 & \varepsilon \\ -1 & 0\end{array}\right](\delta=1)$ or $\left[\begin{array}{cc}1 & 0 \\ 0 & -1\end{array}\right](\delta=-1)$. (Here $\varepsilon$ denotes a generator of the multiplicative group of $F_{q}$.) Also, if $\delta=-1$, then $s$ and $\sigma$ commute, and if $\delta=1$, then $(s w)^{e}$ and $\sigma$ commute and $[\sigma, s] \in\left\langle\left(d_{1} d_{2}\right)^{2^{n}}\right\rangle$.

Proof. We have already seen that $L=O^{2}\left(C_{G}(u)\right)=(B \times O(L))\langle\sigma\rangle$, that $B \cong$ $\operatorname{PSL}(2, q)$ is characteristic in $L$, and that $\langle t, s\rangle$ has the properties of the lemma for every integer $k$. Also, $t$ centralizes $B, s$ centralizes $O(L), s^{2}=[t, s]$ so that $s^{2}$ centralizes $B$, $s$ inverts $\left\langle\left(d_{1} d_{2}\right)^{z}\right\rangle=\left\langle d_{1} d_{2}\right\rangle$ and $w^{s}=w\left(d_{1} d_{2}\right)^{2 k}\left(a_{1} a_{2}\right)^{z}$ for every integer $k$. The argument of [8, Lemma 3.7] now applies to yield (22). Since $\delta p^{f_{1}} \equiv 1\left(\bmod 2^{n}\right)$, let $\delta p^{f_{1}}=1+m 2^{n}$. Then $\sigma^{-1}\left(d_{1} d_{2}\right) \sigma=\left(d_{1} d_{2}\right)^{\delta p_{1}}$ so that $\left[d_{1} d_{2}, \sigma\right]=\left(d_{1} d_{2}\right)^{m 2^{n}}$. Since $z$ normalizes $A=\langle\sigma\rangle$ and $a_{1}, w$ centralize $\sigma$, we have $\sigma^{s}=\sigma\left(d_{1} d_{2}\right)^{k}=\sigma\left(d_{1} d_{2}\right)^{-m k 2^{n}}$ and $\sigma^{s^{2}}=\sigma$ since $s$ inverts $\left(d_{1} d_{2}\right)$. Thus $(B \times O(L))\langle s, t\rangle \triangleleft C_{G}(u)$. But $t$ inverts $O(L)$ and $B$ is simple, hence, $(B \times O(L))\langle s, t\rangle \leqq O^{2 \prime}\left(C_{G}(u)\right)$; therefore,

$$
O^{2 \prime}\left(C_{G}(u)\right)=(B \times O(L))\langle s, t\rangle .
$$

Moreover, when $\delta=-1,[s, \sigma] \in C_{G}(B) \cap B=\{1\}$ so that $[s, \sigma]=1$. When $\delta=1$, then $\sigma^{s w}=\sigma\left(d_{1} d_{2}\right)^{m k 2^{n}}$ so that $\sigma^{(s w) e}=\sigma\left(d_{1} d_{2}\right)^{m k e 2^{n}}=\sigma$, finishing the proof.

A well-known lemma of John Thompson is 
LEMMA 3.10. Let $G$ be a finite group with more than one class of involutions. Let $\alpha, \beta$ be two nonconjugate involutions and let $R$ consist of a set of representatives for the conjugacy classes of involutions of $G$. For $t \in R$, let $a(\alpha, \beta, t)$ denote the number of ordered pairs of involutions $(\sigma, \lambda)$ where $\sigma, \lambda \in C_{G}(t)$ such that $\sigma$ is conjugate in $G$ to $\alpha, \lambda$ is conjugate in $G$ to $\beta$, and such that $t \in\langle(\sigma \lambda)\rangle$. Then

$$
|G|=\left|C_{G}(\alpha)\right|\left|C_{G}(\beta)\right| \sum_{t \in R} \frac{a(\alpha, \beta, t)}{\left|C_{G}(t)\right|} .
$$

We can now prove

LEMMA 3.11. $|G|=|\operatorname{PSp}(4, q)| \rho=\frac{1}{2} q^{4}\left(q^{2}+1\right)\left(q^{2}-1\right) \rho$.

Proof. All involutions of $C_{G}(t)$ and $C_{G}(u)$ are contained in $O^{2 \prime}\left(C_{G}(t)\right)=\left(L_{1} L_{2}\right)\langle u\rangle$ and $O^{2 \prime}\left(C_{G}(u)\right)=(B \times O(L))\langle s, t\rangle$ respectively. Moreover, the structures of $O^{2 \prime}\left(C_{G}(t)\right)$ and $O^{2 \prime}\left(C_{G}(u)\right)$ are completely determined.

It is easy to see that the $G$-fusion of involutions in both $C_{G}(u)$ and $C_{G}(t)$ is determined. Since the semidirect product $H=\langle\bar{\sigma}\rangle$ PSp $(4, q)$ satisfies the hypotheses of the theorem, it follows from the above discussion and Lemmas 2.6 and 3.10 that $|G|=|H|=\rho|\operatorname{PSp}(4, q)|=\frac{1}{2} q^{4}\left(q^{2}+1\right)\left(q^{2}-1\right) \rho$.

4. The $p$-structure of $G$. For $\alpha \in F_{q}$, set

$$
\theta(\alpha)=\left[\begin{array}{ll}
1 & 0 \\
\alpha & 1
\end{array}\right]
$$

Then $\left\{\theta(\alpha) \mid \alpha \in F_{q}\right\}$ is a Sylow $p$-subgroup of SL $\left(2, F_{q}\right)$. Set

$$
\theta_{i}=\phi_{i} \circ \theta: F_{q} \rightarrow L_{i}
$$

and

$$
P_{i}=\text { Image }\left(\theta_{i}\right) \text { for } i=1,2 .
$$

Then $\theta_{i}: F_{q} \rightarrow P_{i}$ is an isomorphism of the additive group of $F_{q}$ onto $P_{i}$ which is a Sylow $p$-subgroup of $L_{\imath}$ for $i=1,2$. Thus $P_{i}$ is elementary Abelian of order $q$ and is normalized by $A=\langle\sigma\rangle$. The subgroup

$$
R=P_{1} P_{2}=P_{1} \times P_{2}
$$

is a Sylow $p$-subgroup of $\mathfrak{S}$. The subgroup

$$
D_{1}=\left\{\theta_{1}(\alpha) \theta_{2}(\alpha) \mid \alpha \in F_{q}\right\}
$$

is a Sylow subgroup of $B$ and is elementary Abelian of order $q$. Set

$$
D_{2}=\left\{\theta_{1}(\alpha) \theta_{2}(-\alpha) \mid \alpha \in F_{q}\right\}
$$

Then

$$
R=P_{1} \times D_{1}=D_{1} \times D_{2}
$$


Put

$$
h=\left[\begin{array}{ll}
\varepsilon & 0 \\
0 & \varepsilon^{-1}
\end{array}\right] \text { and } h_{i}=\phi_{i}(h) \text { for } i=1,2,
$$

where $\varepsilon$ is a generator of the multiplicative group of $F_{q}$. If $\delta=1$, use the same generator $\varepsilon$ as that used in the element $\bar{s}$ of Lemma 3.9. Note that $h=d$ when $\delta=1$, but not when $\delta=-1$. Then

$$
H=\left\langle h_{1}, h_{2}\right\rangle
$$

is an Abelian subgroup of $\mathfrak{S}$ of order $\frac{1}{2}(q-1)^{2}$ and

$$
h_{1}^{q-1 / 2}=h_{2}^{q-1 / 2}=t .
$$

Clearly

$$
N_{G}(R) \cap C_{G}(t)=(R H\langle u\rangle)\langle\sigma\rangle .
$$

Put

$$
\begin{array}{ll}
y=(s w)^{2^{n-2} e} & \text { if } \delta=1, \text { and } \\
y=s^{2^{n-2} e} & \text { if } \delta=-1,
\end{array}
$$

where $s$ is the element of $C_{G}(u)$ referred to in Lemma 3.9. Then

$$
\begin{gathered}
y \in N_{G}\left(D_{1}\right) \text { and }[y, \sigma]=1 . \\
\left(h_{1} h_{2}\right)^{y}=h_{1} h_{2} \text { and } w^{y}=v w \text { if } \delta=1 \text { and } \\
w^{y}=w \text { if } \delta=-1 .
\end{gathered}
$$

Also

$$
y^{2}=u v \text { if } \delta=1 \text { and } y^{2}=u \text { if } \delta=-1
$$

and

$$
t^{y}=t u \text { and }(t u)^{y}=t .
$$

Thus $R^{y}$ is a Sylow p-subgroup of $O^{2 \prime}\left(C_{G}(t u)\right)$ by (38).

Now $D_{1} \leqq R \cap R^{y} \leqq C_{G}(t) \cap C_{G}(t u)=C_{G}(t, u)=(\langle t, u\rangle \times B)\langle\sigma\rangle$. But

$$
R \leqq O^{2 \prime}\left(C_{G}(t)\right)=\mathfrak{B}\langle u\rangle
$$

so that $R \cap R^{y} \leqq O^{2 \prime}\left(C_{G}(t)\right) \cap C_{G}(t, u)=(\mathfrak{S}\langle u\rangle) \cap C_{G}(t, u)=\langle t, u\rangle \times B$. Hence

$$
D_{1}=R \cap R^{y} \text {. }
$$

The proof of $[8$, Lemma 4.1$]$ yields

Lemma 4.1. If $\{1\}<F \leqq O(L)$, then $N_{G}(F) \cap R=N_{G}(F) \cap R^{y}=D_{1}$.

LemMA 4.2. The group $C_{G}\left(D_{1}\right)$ has a normal 2-complement $M$ which is a semidirect product $M=O(L) Q, Q \triangleleft M, O(L) \cap Q=\{1\}$ and where $Q=R^{y} R$ and $|Q|=q^{3}$. 
Proof. It is easy to see that

$$
C_{G}\left(D_{1}\right) \cap C_{G}(u)=\left(D_{1} \times O(L)\right)\left\langle t, s^{2}\right\rangle
$$

and

$$
C_{G}\left(D_{1}\right) \cap C_{G}(t)=R\langle t, u\rangle
$$

Thus

$$
C_{G}\left(D_{1}\right) \cap C_{G}(t u)=R^{y}\langle t, u\rangle .
$$

Now the proof of [8, Lemma 4.2] applies to finish the proof.

Note that $Q$ is characteristic in $M$, which is characteristic in $C_{G}\left(D_{1}\right)$, which is normal in $N_{G}\left(D_{1}\right)$ so that

$$
N_{G}\left(D_{1}\right) \leqq N_{G}(Q) .
$$

LemMA 4.3. The group $Q$ is elementary Abelian of order $q^{3}$ and is the normal 2-complement of $C_{G}(R)$. Also

$$
C_{G}(Q)=Q \text {. }
$$

Proof. From $N_{G}(R) \cap C_{G}(t)=(R H\langle u\rangle)\langle\sigma\rangle$, we conclude that $C_{G}(R) \cap C_{G}(t)$ $=R\langle t\rangle$. Hence $\langle t\rangle$ is a Sylow 2-subgroup of $C_{G}(R)$ so that $C_{G}(R)$ has a normal 2-complement $K$. The four group $\langle t, u\rangle$ normalizes $R$ and hence acts on $K$. Also $C_{K}(t)=R$ and $C_{G}(u) \cap C_{G}(R) \leqq C_{G}(u) \cap C_{G}\left(D_{1}\right)=\left(D_{1} \times O(L)\right)\left\langle t, s^{2}\right\rangle$. But

$$
C_{G}(R) \cap O(L)=\{1\}
$$

by Lemma 4.1 so that $C_{K}(u)=D_{1}$; consequently, $C_{K}(t u) \geqq C_{K}(t, u)=D_{1}$. Assume that $C_{K}(t u) \cap R^{y}=D_{1}$. Then

$$
\begin{aligned}
C_{G}(t u) \cap K & =K \cap C_{G}(t u) \cap C_{G}\left(D_{1}\right)=C_{K}(t u) \cap\left(R^{y}\langle t, u\rangle\right) \\
& =C_{K}(t u) \cap R^{y}=D_{1} .
\end{aligned}
$$

Then [8, Lemma 3.6] implies that $K=R$. The Frattini argument implies that

$$
\begin{aligned}
N_{G}(R) & =C_{G}(R)\left(N_{G}(R) \cap C_{G}(t)\right)=(R\langle t\rangle)\left(N_{G}(R) \cap C_{G}(t)\right) \\
& =N_{G}(R) \cap C_{G}(t)=(R H\langle u\rangle)\langle\sigma\rangle .
\end{aligned}
$$

Hence $R\langle\sigma\rangle$ contains a Sylow p-subgroup of $N_{G}(R)$. However, $Q \cap\langle\sigma\rangle \leqq$ $C_{G}\left(D_{1}\right) \cap\langle\sigma\rangle=\{1\}$ and $\langle\sigma\rangle \leqq N_{G}(Q)$ so that $N_{Q}(R)\langle\sigma\rangle \leqq N_{G}(R)$. Since $N_{Q}(R)>R$, $R\langle\sigma\rangle$ cannot contain a Sylow $p$-subgroup of $N_{G}(R)$ which is a contradiction. Thus $C_{K}(t u) \cap R^{y}>D_{1}$, and the argument of [8, Lemma 4.3] yields $K=Q, R^{y} \leqq C_{G}(R)$ and $C_{G}(Q)=Q$, completing the proof.

Note that $C_{G}(R)=Q\langle t\rangle$ and $N_{G}(R)=(Q\langle t\rangle)\left(N_{G}(R) \cap C_{G}(t)\right)=(Q H\langle u\rangle)\langle\sigma\rangle$. Also $Q$ is characteristic in $C_{G}(R)$ which is normal in $N_{G}(R)$. Hence

$$
N_{G}(R) \leqq N_{G}(Q)
$$


Set

$$
P_{3}=D_{2}^{y} \quad \text { and } \quad \theta_{3}(\alpha)=\left(\theta_{1}(\alpha) \theta_{2}(-\alpha)\right)^{y} \quad \text { for } \alpha \in F_{q} .
$$

Then, by (29) and (35), we have $R^{y}=D_{1} \times P_{3}$ so that

$$
Q=P_{1} \times P_{2} \times P_{3} \text {. }
$$

Moreover, $H \leqq N_{G}(R) \leqq N_{G}(Q)$, and the proof of [8, Lemma 4.4] (provided we observe that $[v, \sigma]=1$ so that replacing $s$ by $s v$, if necessary, is permitted) yields

Lemma 4.4. The action of $H$ on $Q$ is given by

$$
\begin{aligned}
& h_{1}: \theta_{1}(\alpha) \rightarrow \theta_{1}\left(\varepsilon^{2} \alpha\right), \theta_{2}(\alpha) \rightarrow \theta_{2}(\alpha), \theta_{3}(\alpha) \rightarrow \theta_{3}(\varepsilon \alpha), \\
& h_{2}: \theta_{1}(\alpha) \rightarrow \theta_{1}(\alpha), \theta_{2}(\alpha) \rightarrow\left(\varepsilon^{2} \alpha\right), \theta_{3}(\alpha) \rightarrow \theta_{3}(\varepsilon \alpha) .
\end{aligned}
$$

Lemma 4.5. Let $V=O\left(C_{G}\left(P_{1}\right)\right)$. Then $C_{G}\left(P_{1}\right)=L_{2} V, L_{2} \cap V=\{1\}$ and $N_{G}\left(P_{1}\right)$ $=\left(L_{2} V\left\langle h_{1}\right\rangle\right)\langle\sigma\rangle$. The group $V / P_{1}$ is Abelian, $V$ is nilpotent of class at most 2 and $Q \cap V=P_{1} \times P_{3}$ and $\left(L_{2} V\left\langle h_{1}\right\rangle\right) \cap\langle\sigma\rangle=\{1\}$.

Proof. Clearly $N_{G}\left(P_{1}\right) \cap C_{G}(t)=\left(P_{1}\left\langle h_{1}\right\rangle L_{2}\right)\langle\sigma\rangle$ so that $C_{G}\left(P_{1}\right) \cap C_{G}(t)=P_{1} L_{2}$. The proof of [8, Lemma 4.5] applies to give all but the last equation of the lemma.

Finally, by considering the action of an element of $\left(C_{G}\left(P_{1}\right)\left\langle h_{1}\right\rangle\right) \cap\langle\sigma\rangle$ on $P_{1}$, we have $\left(C_{G}\left(P_{1}\right)\left\langle h_{1}\right\rangle\right) \cap\langle\sigma\rangle \leqq C_{G}\left(P_{1}\right) \cap\langle\sigma\rangle=1$. This completes the proof.

Lemma 4.6. $C_{G}\left(P_{3}\right)$ has a normal 2-complement, and $O\left(C_{G}\left(P_{3}\right)\right) \leqq Q H$.

Proof. If $\delta=1$, then $a_{2}^{2^{n-2}}: D_{1} \rightarrow D_{2}$, so that $C_{G}\left(D_{2}\right)$ has a normal 2-complement by Lemma 4.2. If $\delta=-1$, then $N_{G}\left(D_{2}\right) \cap C_{G}(t)=\left(R\left\langle h_{1} h_{2}\right\rangle\langle t, u\rangle\right)\langle\sigma\rangle$ implies that $C_{G}\left(D_{2}\right) \cap C_{G}(t)=R\langle t\rangle$. Thus $\langle t\rangle$ is a Sylow 2-subgroup of $C_{G}\left(D_{2}\right)$ so that $C_{G}\left(D_{2}\right)$ has a normal 2-complement. Since $P_{3}=D_{2}^{y}, C_{G}\left(P_{3}\right)$ always has a normal 2-complement. In either case, $\langle t, u\rangle \leqq N_{G}\left(D_{2}\right)$ so that $\langle t, u\rangle$ acts on $O\left(C_{G}\left(D_{2}\right)\right)$. From the above, we have $O\left(C_{G}\left(D_{2}\right)\right) \cap C_{G}(t)=R$. Since $D_{1} \leqq B \triangleleft C_{G}(u)$ and every pair of distinct Sylow $p$-subgroups of $B$ generates $B$, every odd ordered subgroup of $C_{G}(u)$ containing $D_{1}$ is contained in $N_{G}\left(D_{1}\right) \cap C_{G}(u)$. By Lemma 3.9, this group has a normal 2-complement $\langle\sigma\rangle F O(L)$ where $F=D_{1}\left\langle\left(h_{1} h_{2}\right)^{2^{m}}\right\rangle$ is the normal 2-complement of $N_{B}\left(D_{1}\right)$. If $C_{G}\left(D_{2}\right)$ contains an element of form $\sigma^{i} x z$ where $x \in F$ and $z \in O(L)$, then $\left[t, \sigma^{i} x z\right]=z^{2} \in C_{G}\left(D_{2}\right)$. By Lemma $4.1, z^{2}=1$ so that $z=1$. Hence $O\left(C_{G}\left(D_{2}\right)\right) \cap C_{G}(u) \leqq\langle\sigma\rangle F$. However, no nontrivial element of form $\left(h_{1} h_{2}\right)^{j} \sigma^{i}$ centralizes $D_{2}$. Hence $O\left(C_{G}\left(D_{2}\right)\right) \cap C_{G}(u)=D_{1}$. Since $D_{1}$ is normalized by $\langle\sigma\rangle$, there exists a maximal odd order subgroup $X$ of $C_{G}(t)$ containing $D_{1}$ which is normalized by $\langle\sigma\rangle$. Thus $X \leqq \mathfrak{S}\langle\sigma\rangle$ and $X=(X \cap \mathfrak{S})\langle\sigma\rangle$. But $D_{1} \leqq X \cap \mathfrak{S}$ and $R H$ contains a unique largest odd order subgroup of $\mathfrak{S}$ containing $D_{1}$ and hence $X \leqq R H\langle\sigma\rangle$. Since $\langle\sigma\rangle \leqq N_{G}\left(D_{2}^{y^{-1}}\right)$ and $D_{1} \leqq O\left(C_{G}\left(D_{2}^{y-1}\right)\right) \cap C_{G}(t)$, we conclude that $O\left(C_{G}\left(D_{2}^{y-1}\right)\right) \cap C_{G}(t) \leqq R H\langle\sigma\rangle$, so that $O\left(C_{G}\left(D_{2}\right)\right) \cap C_{G}(t u) \leqq R^{y} H^{y}\langle\sigma\rangle$. Hence $O\left(C_{G}\left(D_{2}\right)\right) \leqq D_{1} R R^{y} H^{y}\langle\sigma\rangle=Q H^{y}\langle\sigma\rangle$. Thus $O\left(C_{G}\left(P_{3}\right)\right) \leqq Q H\langle\sigma\rangle$. Since no element of $H\langle\sigma\rangle-H$ centralizes $P_{3}, O\left(C_{G}\left(P_{3}\right)\right) \leqq Q H$. 
Lemma 4.7. $V=O\left(C_{G}\left(P_{1}\right)\right)$ is a p-group and $V=P_{1} P_{3} P_{4}$ where $P_{4}=P_{3}^{c_{2}}$ with $c=\left[\begin{array}{cc}0 & -1 \\ 1 & 0\end{array}\right]$ and $c_{2}=\phi_{2}(c)$.

Proof. Now

$$
\begin{aligned}
C_{V}\left(P_{3}\right) & \leqq V \cap O\left(C_{G}\left(P_{3}\right)\right) \\
& =V \cap O\left(C_{G}\left(P_{3}\right)\right) \cap Q H \cap C_{G}\left(P_{1}\right)=V \cap O\left(C_{G}\left(P_{3}\right)\right) \cap\left(Q\left\langle h_{2}\right\rangle\right) \leqq V \cap Q \\
& =P_{1} \times P_{3} \leqq C_{V}\left(P_{3}\right)
\end{aligned}
$$

so that $C_{V}\left(P_{3}\right)=P_{1} \times P_{3}$ which is a $p$-group. Since $V$ is nilpotent, $V$ is a $p$-group. Also $c_{2} \in C_{G}(t) \cap C_{G}\left(P_{1}\right)$ so that $P_{4}=P_{3}^{c_{2}} \leqq V$. Since $h_{1} h_{2}^{-1} \in C_{G}\left(P_{3}\right)$, we have $\left(h_{1} h_{2}^{-1}\right)^{c_{2}}=h_{1} h_{2} \in C_{G}\left(P_{4}\right)$. But $h_{1} h_{2}$ acts fixed point free on $P_{1} \times P_{3}$ by Lemma 4.4; hence $P_{1} P_{3} \cap P_{4}=\{1\}$. We have seen that $P_{1} \geqq V^{\prime}$ so $P_{1} P_{3} P_{4}$ is a subgroup of $V$ of order $q^{3}$. But $\left|P_{2} V\langle\sigma\rangle\right|=q \rho|V|$ so that $|V| \leqq q^{3}$ by Lemma 3.11. Thus $V=P_{1} P_{3} P_{4}$ which completes the proof of the lemma.

Note that $\left[\sigma, c_{2}\right]=1$ so that if

$$
\theta_{4}(\alpha)=\theta_{3}(\alpha)^{c_{2}} \text { for } \alpha \in F_{q},
$$

then

$$
\theta_{4}(\alpha)^{\sigma}=\left(\theta_{3}(\alpha)^{\sigma}\right)^{c_{2}}=\theta_{3}\left(\alpha^{\bar{\sigma}}\right)^{c_{2}}=\theta_{4}\left(\alpha^{\bar{\sigma}}\right) \text { for } \alpha \in F_{q} .
$$

Also $c_{2}$ centralizes $h_{1}$ and inverts $h_{2}$ so that

and

$$
\theta_{4}(\alpha)^{h_{1}}=\theta_{4}(\varepsilon \alpha) \text { and } \theta_{4}(\alpha)^{h_{2}}=\theta_{4}\left(\varepsilon^{-1} \alpha\right) \text { for } \alpha \in F_{q}
$$

$H\langle\sigma\rangle \leqq N_{G}\left(P_{4}\right)$

Lemma 4.8. If $U=P_{2} V=P_{1} P_{2} P_{3} P_{4}$, then $N_{G}(U) \cap N_{G}\left(P_{1}\right)=U H\langle\sigma\rangle \leqq N_{G}(Q)$. Also $(U H) \cap\langle\sigma\rangle=\{1\}, P_{1} P_{3} \triangleleft U$ and $U / P_{1} P_{3}$ is Abelian.

Proof. Since $U=Q P_{4}$, we have $N_{P_{4}}(Q)>\{1\}$. But if $\alpha \in F_{q}$, then $\theta_{4}(\alpha)^{h_{1}}=$ $\theta_{3}(\alpha)^{c_{2} h_{1}}=\theta_{3}(\alpha)^{h_{1} c_{2}}=\theta_{4}(\varepsilon \alpha)$ by Lemma 4.4 and $h_{1} \in N_{G}(Q)$. Thus $\left\langle h_{1}\right\rangle$ normalizes $N_{P_{4}}(Q)$ and acts irreducibly on $P_{4}$. Consequently, $N_{P_{4}}(Q)=P_{4}$ so $Q \triangleleft U$. Then

$$
\begin{aligned}
N_{G}(U) \cap N_{G}\left(P_{1}\right) & =N_{G}(U) \cap\left(\left(L_{2} V\left\langle h_{1}\right\rangle\right)\langle\sigma\rangle\right)=\left(\left(N_{G}(U) \cap L_{2}\right) V\left\langle h_{1}\right\rangle\right)\langle\sigma\rangle \\
& =\left(\left(N_{G}\left(P_{2}\right) \cap L_{2}\right) V\left\langle h_{1}\right\rangle\right)\langle\sigma\rangle=U H\langle\sigma\rangle \leqq N_{G}(Q) .
\end{aligned}
$$

Clearly $P_{1} \leqq Z(U)$ and $U H\langle\sigma\rangle \leqq N_{G}\left(P_{1}\right)$. By considering the action of $U H\langle\sigma\rangle$ on $P_{1}$, we conclude that $U H \cap\langle\sigma\rangle=\{1\}$. Also $P_{1} P_{3}=Q \cap V \triangleleft U$ and $U=Q V$. However, $[Q, V] \leqq Q \cap V=P_{1} \times P_{3}$ and $Q / P_{1} P_{3}$ and $V / P_{1} P_{3}$ are Abelian, consequently $U / P_{1} P_{3}$ is Abelian.

5. The $(B N)$-pair. The proof of [8, Lemma 5.1] yields

LEMMA 5.1 .

and

$$
u: \theta_{1}(\alpha) \rightarrow \theta_{2}(\alpha), \theta_{2}(\alpha) \rightarrow \theta_{1}(\alpha), \theta_{3}(\alpha) \rightarrow \theta_{3}(-\alpha)
$$

$$
c_{2}: \theta_{1}(\alpha) \rightarrow \theta_{1}(\alpha), \theta_{3}(\alpha) \rightarrow \theta_{4}(\alpha), \theta_{4}(\alpha) \rightarrow \theta_{3}(-\alpha)
$$

for all $\alpha \in F_{q}$. 
Since $\langle t\rangle$ is characteristic in $H, N_{G}(H)=N_{G}(H) \cap C_{G}(t)=\left\langle H, u, c_{2}\right\rangle\langle\sigma\rangle$. Set

$$
N=(\mathfrak{S}\langle u\rangle) \cap N_{G}(H)=\left\langle H, u, c_{2}\right\rangle .
$$

Clearly $\langle\sigma\rangle$ normalizes $N$.

LEMMA 5.2. The structure of $N=\left\langle H, u, c_{2}\right\rangle$ is determined by the relations: $h_{1}^{q-1 / 2}=h_{2}^{q-1 / 2}=c_{2}^{2}=t, t^{2}=u^{2}=1,\left[h_{1}, h_{2}\right]=\left[h_{1}, c_{2}\right]=1, h_{1}^{u}=h_{2}, h_{2}^{c}=h_{2}^{-1},\left(u c_{2}\right)^{4}=1$. The group $W=N / H$ is dihedral of order 8 .

The proof of this lemma is the same as that of [8, Lemma 5.2].

Set $r_{1}=u H$ and $r_{2}=c_{2} H$; then $r_{1}, r_{2}$ are involutions which generate $W=N / H$. The elements of $W$ written in shortest possible form in terms of $r_{1}$ and $r_{2}$ are as given in [8, p. 30]. For $\alpha \in W$, let $\lambda(\alpha)$ be the numbers of factors $r_{i}$ when $\alpha$ is expressed in its shortest form. Also define $\omega: W \rightarrow N$ as in [8, p. 30] so that $\alpha=\omega(\alpha) H$ for all $\alpha \in W$. If $K$ is any subgroup of $G$ containing $H$, write $\alpha K$ and $K \alpha$ for the cosets $\omega(\alpha) K$ and $K \omega(\alpha)$ respectively. If $K$ is any subgroup normalized by $H$, write $K^{\alpha}$ for $K^{\omega(\alpha)}$. Set

$$
\mathfrak{B}=U H \text {. }
$$

Clearly $\mathfrak{B} \cap N=H$.

The proofs of the following two lemmas are the same as those of [8, Lemmas 5.3 and 5.4].

Lemma 5.3. Let $G_{i}=\mathfrak{B} \cup \mathfrak{B} r_{i} \mathfrak{B}$ for $i=1,2$. Then $G_{1}$ and $G_{2}$ are subgroups of $G$.

Lemma 5.4. If $\alpha \in W$ and $\lambda\left(r_{i} \alpha\right) \geqq \lambda(\alpha)$ for $i=1$ or 2 , then $r_{i} \mathfrak{B} \alpha \subseteq \mathfrak{B} r_{i} \alpha \mathfrak{B}$.

Lemma 5.5. Set $G_{0}=\mathfrak{B N}$. Then $G_{0}$ is a subgroup of $G$, and $G_{0}$ is the disjoint union of the eight double cosets $\mathfrak{B} \alpha \mathfrak{B}$ for $\alpha \in W$. Also $\langle\sigma\rangle \leqq N_{G}(\mathfrak{B}) \cap N_{G}(N) \leqq N_{G}\left(G_{0}\right)$ and $G_{0} \cap\langle\sigma\rangle=\{1\}$.

Proof. The first statement follows from Lemmas 5.3 and 5.4 by a theorem of Tits [7]. Since the second statement is already known, it remains to prove that $G_{0} \cap\langle\sigma\rangle=\{1\}$. But $G_{0}=U N U$ and $N \cap(U\langle\sigma\rangle)=N \cap C_{G}(t) \cap(U\langle\sigma\rangle)=N \cap(R\langle\sigma\rangle)$ $=\{1\}$ by the structure of $C_{G}(t)$ and the lemma follows.

Using our knowledge of the action of $\mathrm{H}_{\text {on }} \mathrm{P}_{4}$ as given in (50), the same proof of $[8$, Lemma 5.6] yields

LEMMA 5.6. $U \cap U^{r_{1} r_{2} r_{1} r_{2}}=\{1\}$.

Similarly the arguments of [8, Lemmas 5.7 and 5.8] yield

LEMMA 5.7. $\left|G_{0}\right|=|\operatorname{PSp}(4, q)|$.

LEMma 5.8. $G_{0} \cong \operatorname{PSp}(4, q), G_{0} \triangleleft G_{0}\langle\sigma\rangle,\langle\sigma\rangle \cap G_{0}=\{1\}$ and $O(G)=\{1\}=C_{G}\left(G_{0}\right)$. Finally $G$ is isomorphic to the semidirect product $\langle\bar{\sigma}\rangle \operatorname{PSp}(4, q)$ described in the introduction. 
Proof. By Lemmas 5.5, 5.7 and 3.11, we have that $G=G_{0}\langle\sigma\rangle \triangleright G_{0}$. We now prove that $G_{0} \cong \operatorname{PSp}(4, q)$.

Clearly $G_{0}$ contains $\left\langle P_{2}, P_{2^{2}}^{c_{2}}\right\rangle=L_{2}$ and hence $G_{0}$ contains $L_{1}=L_{2}^{u}$. Thus $C_{G}(t) \cap G_{0}$ $=\left(L_{1} L_{2}\right)\langle u\rangle$ by Lemma 5.5. Since $\left|G / G_{0}\right|$ is odd, $t$ and $u v$ are involutions in $G_{0}$ which are fused in $G_{0}$ by Lemma 2.6. Now [8] implies that $G_{0} \cong \operatorname{PSp}(4, q)$. The rest follows easily.

\section{REFERENCES}

1. R. W. Carter, Simple groups and simple Lie algebras, J. London Math. Soc. 40 (1965), 193-240. MR 30 \#4855.

2. P. Fong and W. J. Wong, A characterization of the finite simple groups $\mathrm{PSp}(4, q), G_{2}(q)$, $D_{4}^{2}(q)$. I, Nagoya Math. J. 36 (1969), 143-184. MR 41 \#326.

3. D. Gorenstein and J. H. Walter, On finite groups with dihedral Sylow 2-subgroups, Illinois J. Math. 6 (1962), 553-593. MR 26 \#188.

4. - The characterization of finite groups with dihedral Sylow 2-subgroups. I, J. Algebra 2 (1965), 85-151. MR 31 \#1297.

5. B. Huppert, Endliche Gruppen. I. Die Grundlehren der math. Wissenschaften, Band 134, Springer-Verlag, Berlin and New York, 1967. MR 37 \#302.

6. D. S. Passman, Permutation groups, Benjamin, New York, 1968. MR 38 \#5908.

7. J. Tits, Théorème de Bruhat et sous-groupes paraboliques, C. R. Acad. Sci. Paris 254 (1962), 2910-2912. MR 25 \#2149.

8. W. J. Wong, A characterization of the finite projective symplectic groups $\operatorname{PSp}_{4}(q)$, Trans. Amer. Math. Soc. 139 (1969), 1-35. MR 40 \#5724.

Department of Mathematics, University of Illinois at Chicago Circle, Chicago, ILLINOIS 60680 